Bundesgesundheitsbl 2020 - 63:1331-1340 https://doi.org/10.1007/s00103-020-03222-8 Eingegangen: 11 . Mai 2020

Angenommen: 7. September 2020 Online publiziert: 6 . Oktober 2020 (c) Der/die Autor(en) 2020

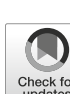

Alexander Michels - Jessica Hartmann - Christian J. Buchholz

Paul-Ehrlich-Institut, Langen, Deutschland

\title{
Chimäre Antigenrezeptoren (CARs) in der Onkologie: eine Übersicht zu klinischer Anwendung und neuen Entwicklungen
}

ren (engl. Chimeric Antigen Receptors, CARs).

So konnten bemerkenswerte klinische Erfolge bei der Behandlung von Patienten mit hämatologischen Krebserkrankungen durch die Gabe von CAR-T-Zellen erzielt werden. Die CARs sind dabei gegen das B-zellspezifische Antigen Cluster of Differentiation 19 (CD19) gerichtet. Dies führte 2018 zur Zulassung von zwei CAR-T-Zellprodukten in Europa, nämlich Kymriah (Tisagenlecleucel, Novartis) und Yescarta (Axicabtagene ciloleucel, Kite). Die positiven klinischen Befunde wurden jedoch von Berichten über schwere Nebenwirkungen begleitet. So gab es Fälle von Zytokinfreisetzungssyndrom (engl. Cytokine Release Syndrome, CRS) und Neurotoxizität, einige Patienten verstarben [4].

Inzwischen haben Studien mit spezialisierten Mausmodellen wertvolle Hinweise zum Zustandekommen von CRS und Neurotoxizität geliefert [5-8]. In diesem Übersichtsartikel besprechen wir die verwendeten Ansätze und ihre Implikationen für die CAR-Therapie. Des Weiteren zeigen wir mögliche Wege auf, die logistischen und wirtschaftlichen Hürden beim Einsatz von CAR-Therapien zu umgehen, z. B. durch eine Automatisierung der Herstellung von CAR-Zellen.

\section{Was sind CAR-Zellen? Wie werden sie erzeugt?}

CARs sind aus mehreren Domänen verschiedener Immunrezeptoren zu- sammengesetzt, die einen synthetischen, chimären Rezeptor bilden (• Abb. 1a). Ein Transmembranprotein mit einem einzigen Membrandurchgang präsentiert an $\mathrm{N}$-terminaler Position die Antigenbindedomäne. Im Fall der zugelassenen CAR-Zellprodukte handelt es sich dabei um ein Antikörpereinzelkettenfragment (engl. Single Chain Variable Fragment, scFv), ein aus den variablen Teilen der schweren und leichten Kette eines Immunglobulins bestehendes Fusionsprotein, das die Antigenspezifität des CAR bestimmt. CARs können auch mit anderen Arten von Bindedomänen konstruiert werden, zum Beispiel solchen auf Basis von Nanobodies, Fab-Fragmenten oder Designed Ankyrin Repeat Proteins (DARPins; [9-11]). In C-terminaler Richtung folgen auf die Antigenbindedomäne dann eine Scharnierregion (engl. Hinge) und die Transmembrandomäne. Obwohl die genauen Signaltransduktionsmechanismen von CARs noch nicht ausreichend verstanden sind, gibt es Hinweise auf die Wichtigkeit der Wahl von Scharnier- und Transmembrandomäne für eine optimale Signaltransduktion [10, 12]. Auf intrazellulärer Seite befinden sich Signaldomänen, die bei Bindung des Antigens das Signal weiterleiten. CARs der sog. zweiten Generation enthalten dabei die Signaldomäne des

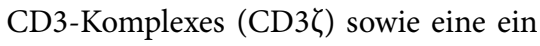
kostimulatorisches Signal auslösende Domäne, z.B. von CD28 oder 4-1BB (CD137). Komplexere CARs können zusätzliche Signaldomänen („dritte Ge- 


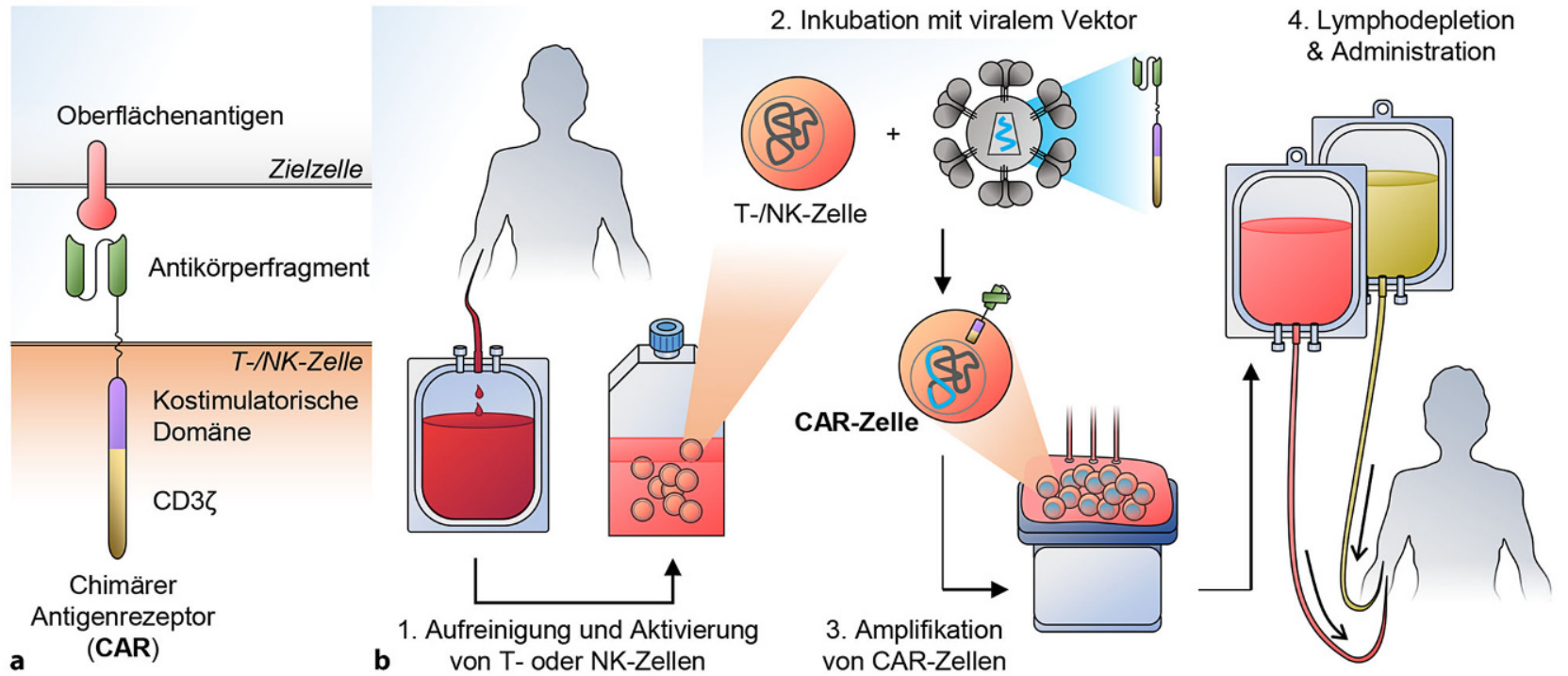

Abb. $1 \Delta$ a Architektur eines chimären Antigenrezeptors (CAR). Die Antigenbindedomäne, hier ein Einzelkettenantikörperfragment, bindet Epitope nativer Strukturen auf der Oberfläche der Zielzelle. Bei Antigenbindung lösen die intrazellulären (ko)stimulatorischen Domänen ein Aktivierungssignal in derCAR-Immunzelle aus, welches zurZerstörung der Zielzellen führt. b Herstellung eines autologen CAR-Zellprodukts. Aus einer Patientenblutspende werden T-oder NK-Zellen isoliert und ex vivo kultiviert. Durch Transduktion mit z. B. lentiviralen Vektoren wird ein für den CAR codierendes Gen ins Erbmaterial der Zellen integriert. So generierte CAR-Zellen werden dann weiter vermehrt, bevor sie, folgend auf eine lymphodepletierende Vorbehandlung, in den Patienten reinfundiert werden. Für die Herstellung eines allogenen CAR-Zellprodukts werden die T-oder NK-Zellen aus Fremdblutspenden gewonnen. Adaptiert aus [4]

neration") enthalten oder Strukturen, die ein Zytokinsignal hervorrufen („vierte Generation“, etwa TRUCKs; $[4,12,13])$.

CARs können sowohl in T-Zellen als auch in natürliche Killerzellen (NK-Zellen) eingebracht werden und bewirken in diesen bei Antigenbindung eine Verschiebung der Signalverhältnisse zugunsten der Aktivierung der Zellen gegenüber Tumorzellen. Die Aktivierung von T-Zellen ist, u.a. zur Vermeidung von $\mathrm{Au}-$ toimmunreaktionen, ein streng regulierter Prozess. Wegen der subtilen bzw. transienten molekularen Unterschiede zwischen Krebszellen und gesunden Körperzellen und des Selbstursprungs von Tumorzellen kommt es häufig zu keiner (effizienten) Antitumorreaktion des Immunsystems. Kritische Komponenten der Aktivierung von T-Zellen, sonst zwischen 2 Rezeptorkomplexen aufgeteilt, liegen bei CAR-T-Zellen unter der Kontrolle eines einzigen Signals, der Erkennung des Antigens durch die Antigenbindedomäne des CAR. So werden die kanonischen Immunsignalachsen, nämlich T-Zellrezeptor und Kostimulation (z. B. CD28-CD80), die durch ihre komplexe
Regulation Tumortoleranz begünstigen können, umgangen. Ein weiterer Unterschied zu normaler T-Zellfunktion besteht in der Erkennung nativer Antigene durch den CAR, im Gegensatz zu proteolytisch prozessierten, durch Major Histocompatibility Complexes (MHCs) präsentierten Peptidantigenen, welche von T-Zellrezeptoren erkannt werden [14].

Zusätzlich zu CAR-T-Zellprodukten werden aktuell auch auf NK-Zellen basierende Krebstherapien in klinischen Studien evaluiert. NK-Zellen agieren als Brücke zwischen angeborenem und adaptivem Immunsystem. Sie werden - im Gegensatz zu T-Zellen - nicht über die MHC-TCR-Achse aktiviert, sondern integrieren Signale aus einem begrenzteren, in der Keimbahn codierten Repertoire polymorpher inhibitorischer und aktivierender Rezeptoren [15]. Erste klinische Studien haben gezeigt, dass nur teilweise mit EmpfängerMHC kompatible, allogene NK-Zellen in Kombination mit lymphodepletierender Vorbehandlung sicher und effektiv zur Behandlung von Tumorerkrankungen eingesetzt werden können [16]. Sie sind außerdem interessant für CAR-Therapien, weil sie zytotoxisch wirken und dabei CAR-mediierte und CAR-unabhängige Antitumoraktivität zeigen können. NKZellen sind mit den gleichen CARArchitekturen kompatibel wie T-Zellen.

Wenngleich die Prozesse zur Herstellung von in klinischen Studien eingesetzten CAR-Zellprodukten im Detail recht heterogen sind, gleichen sie sich in ihren grundlegenden Schritten. Die zur Herstellung eines autologen CAR-Therapieprodukts notwendigen Arbeitsschritte sind in $\mathbf{D A b b} \mathbf{1 b}$ illustriert. Die relevanten Immunzellen ( $\mathrm{T}$ - oder NK-Zellen) werden aus dem Blut des Patienten isoliert und außerhalb des Körpers (ex vivo) kultiviert. Zu geeigneten Zeitpunkten werden das Wachstum und Überleben der Zellen begünstigende Biomoleküle (wie agonistische Antikörper, Interleukine) zugegeben. Darauffolgend wird das für den CAR codierende genetische Material in die Zellen eingebracht. Meistens werden dafür retrovirale oder vom humanen Immundefizienzvirus 1 (HIV-1) abgeleitete lentivirale Vektoren verwendet. Retro- und lentivirale Vektoren integrie- 
ren die CAR-enthaltende Genkassette in das chromosomale Material der Zellen. Die resultierenden CAR-Zellen werden dann für einige Tage in Zellkultur amplifiziert, bevor das Zellmaterial, meist nach einer vorbereitenden Behandlung des Patienten mit Chemotherapeutika, reinfundiert wird $[17,18]$.

\section{CAR-Zellen in der klinischen Anwendung}

Die beiden zugelassenen Arzneimittel, Axicabtagene ciloleucel und Tisagenlecleucel, sind derzeit die am häufigsten in der Klinik angewendeten CAR-TZellprodukte. In der für die Zulassung ausschlaggebenden Studie ZUMA-1 zur Behandlung von aggressivem NonHodgkin-Lymphom in Patienten, bei denen Standardtherapien versagt hatten, erzielte Axicabtagene ciloleucel eine mit $66 \%$ mehr als doppelt so hohe Ansprechrate wie bei der historischen Kontrolle [19]. Für die Indikation akute lymphatische Leukämie (ALL) erreichten in der zulassungsrelevanten Studie von 75 mit Tisagenlecleucel behandelten Probanden über $80 \%$ vollständige Remission und über $75 \%$ überlebten für mindestens 12 Monate nach der Behandlung. Beide Ergebnisse waren besser als die der herangezogenen Chemotherapieund Antikörpervergleichstherapien [20]. Weniger eindeutig waren die Daten zur Behandlung des diffusen großzelligen B-Zell-Lymphoms (DLBCL). Aufgrund der langen Zeit zwischen Patienteneinschluss und CAR-T-Zellinjektion (54 Tage) kam es hier zu einem ungewöhnlich hohen Anteil an Patienten, welche die Teilnahme an der Studie abbrachen. Ausschlaggebend für das positive Votum bei der Europäischen Arzneimittel-Agentur (EMA) waren dann u.a. die lang anhaltenden Effekte bei den Patienten, bei denen die Therapie angeschlagen hatte [20].

Seit den Zulassungserteilungen sind in der EU bis September 2019 mehr als 100 Patienten mit den kommerziellen Produkten behandelt worden [21]. Alle Patienten werden im Melderegister der European Society for Blood and Marrow Transplantation dokumentiert (www.EBMT.org). Außerdem erfolgen

Bundesgesundheitsbl 2020 • 63:1331-1340 https://doi.org/10.1007/s00103-020-03222-8

(c) Der/die Autor(en) 2020

A. Michels · J. Hartmann · C. J. Buchholz

Chimäre Antigenrezeptoren (CARs) in der Onkologie: eine
Übersicht zu klinischer Anwendung und neuen Entwicklungen

\section{Zusammenfassung}

2018 erhielten 2 neuartige Krebstherapien auf Basis chimärer Antigenrezeptoren (CARs) die Marktzulassung in der Europäischen Union. Die Produkte, zunächst zugelassen für die Bekämpfung weit fortgeschrittener Leukämien bzw. Lymphome, erhielten nicht nur wegen ihrer neuen Wirkungsweise und Behandlungserfolge viel Aufmerksamkeit, sondern auch wegen ihrer teilweise gravierenden Nebenwirkungen sowie der wirtschaftlichen und logistischen Herausforderungen, die mit ihrer Herstellung verknüpft sind. Nun, fast 2 Jahre später, belegen Hunderte laufende klinische Studien das weltweite Bestreben, das Potenzial der CAR-Technologie voll zu erschließen. Dazu gilt es, die Effektivität der Behandlung bei verschiedenen Krankheitsbildern sicherzustellen, das Nebenwirkungsprofil zu verstehen und zu verbessern und die Herstellung des Zellpräparats robuster zu gestalten.

In diesem Artikel beschreiben wir die

Studienlage sowie das Wirkprinzip von
CAR-T- und -NK-Zellen. In jüngster Zeit hat eine Reihe von Studien in fortgeschrittenen Tiermodellen Einblicke in die potenziellen Ursachen schwerer Nebenwirkungen der CAR-Therapie ermöglicht. Wir fassen diese Ergebnisse zusammen und erläutern die Funktionsweise verfügbarer Tiermodelle. Zusätzlich zeigen wir mögliche weitere Ansätze auf, die momentanen Limitationen der Technologie zu umgehen und sie breit und sicher einsetzbar zu machen. Bisher als letztes Mittel bei sehr schweren Krankheitsverläufen eingesetzt, scheint die CAR-Therapie am Beginn einer Entwicklung hin zu einem neuen Konzept bei der Behandlung eines breiten Spektrums von hämatologischen und soliden Tumorerkrankungen zu stehen.

Schlüsselwörter

Hämatologische Tumorerkrankungen . Immuntherapie · Genetisch modifizierte Zellen · ATMP.CAR

\section{Chimeric antigen receptors in oncology: clinical applications and new developments}

\section{Abstract}

In 2018, two novel cancer therapies based on chimeric antigen receptors (CARs) were granted marketing authorization in the European Union. Authorized for use against advanced lymphoma and/or leukemia, the products were at the center of international attention, not only due to their novel mode of action and their encouraging efficacy but also because of their sometimes severe side effects and the economic and logistic challenges posed by their manufacture. Now, almost two years later, hundreds of active clinical trials emphasize the global drive to harness the full potential of CAR technology. In this article, we describe the mode of action of CAR T and CAR NK cells and review the clinical testing situation as well as early real- world data. In recent years, preclinical studies using advanced animal models have provided first insights into the mechanisms underlying the severe side effects of CAR T therapy. We summarize their results and describe the available models. Additionally, we discuss potential solutions to the hurdles currently limiting CAR technology. So far used as lastline treatment for patients with aggressive disease, CAR technology has the potential to become a new, broadly effective standard for tumor therapy.

\section{Keywords}

Hematologic malignancies · Immunotherapy . Genetically modified cells $\cdot$ ATMP $\cdot$ CAR
Nachbeobachtungsstudien, mit denen bei der Zulassung erhaltene Auflagen erfüllt werden. Sobald vollständig, werden die nach Zulassung gewonnenen Wirksamkeitsdaten interessante Vergleiche mit den zur Zulassung vorgelegten
Daten ermöglichen. Erste Analysen für Axicabtagene ciloleucel aus dem kommerziellen Anwendungsbetrieb in der Behandlung von Non-Hodgkin-Lymphomen [22, 23] zeigen im Vergleich mit der ZUMA-1 Studie ein geringfügig 


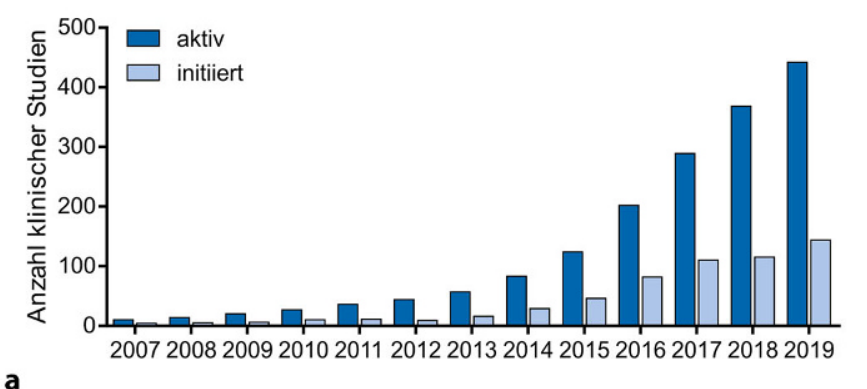

a
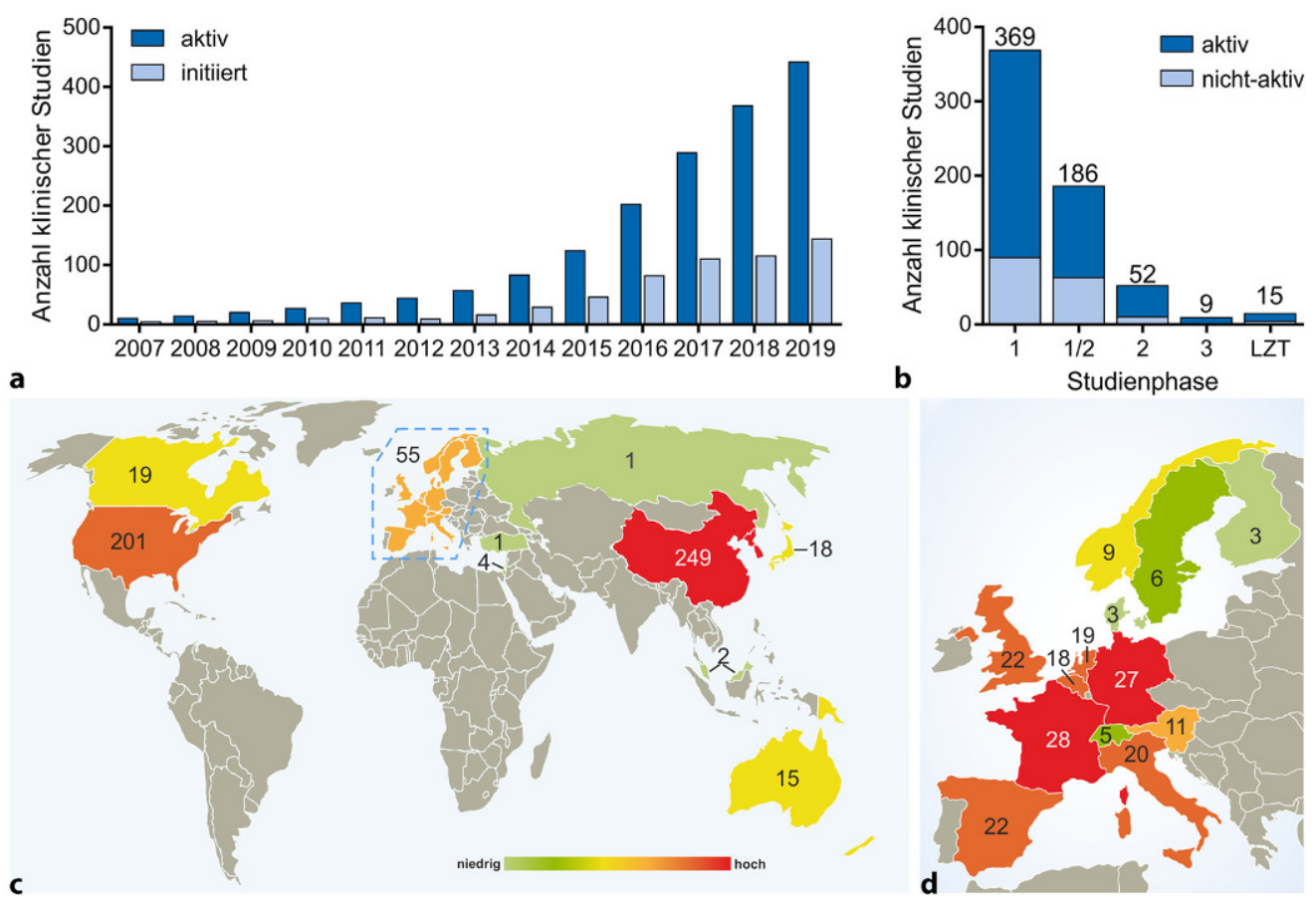

Abb. $2 \Delta$ Erhebungen zu CAR-T-Zellstudien. Die Angaben wurden dem freiwilligen US-Register clinicaltrials.gov mithilfe des in [4] beschriebenen Algorithmus entnommen. a CAR-T-Zellstudien im zeitlichen Verlauf ab 2007 mit Anzahl der weltweit pro Jahr initiierten Studien sowie Gesamtzahl der laufenden (aktiven) Studien bis Ende 2019. b Verteilung von aktiven und nichtaktiven CAR-T-Zellstudien auf die verschiedenen klinischen Studienphasen. Angegeben ist die Gesamtzahl der Studien in der jeweiligen Studienphase. Keine Angabe bei 43 Studien. LZT, Langzeitstudie. c Weltweite geografische Verteilung von laufenden CAR-T-Zellstudien. In d ist eine detaillierte Aufteilung der Studien für die gekennzeichnete Region gezeigt. Der Farbcode gibt die Prävalenz der Studienanzahl von niedrig (grün) bis hoch (rot) an. Die exakte Anzahl der laufenden CAR-T-Zellstudien pro Land ist angegeben. Bei 11 Studien handelt es sich um Langzeitstudien. 36 Studien weltweit und 29 innerhalb von Europa haben multinationale Beteiligung und sind mehrfach repräsentiert. Keine Angabe zu beteiligten Studienzentren bei 25 Studien

schlechteres Ansprechen [24]. Allerdings werden hier insbesondere die Langzeiteffekte zu bewerten sein, zu denen derzeit noch keine abschließenden Aussagen getroffen werden können [21].

Neben der klinischen Anwendung der zugelassenen CAR-T-Zellen finden weltweit viele klinische Studien statt, in denen neuentwickelte CARs getestet oder bereits zugelassene unter neuen Bedingungen eingesetzt werden. Auf Basis des freiwilligen US-Registers clinicaltrials.gov [25] lässt sich die Dynamik der Studienlage analysieren. Zum Anfang des zweiten Quartals 2020 sind 675 CAR-TStudien für Tumortherapien registriert, darunter 15 Langzeitstudien. Demnach stieg die Zahl aktiver Studien seit 2016 um mehr als $100 \%$ auf 495 Studien weltweit, darunter 11 Langzeitstudien. 92 Studien sind bereits beendet. Die Anzahl aktiver Studien steigt also seit 2014 kontinuierlich an und hat sich seitdem knapp verfünffacht (• Abb. 2a).

Die meisten CAR-T-Studien laufen in China und den USA (• Abb. 2c). Seit 2017 hat sich die Zahl klinischer Studien dort etwa verfünffacht bzw. verdoppelt. Weniger als $10 \%$ (36 von 495 Studien) der weltweit laufenden CAR-T-Studien sind multinational angelegt. Dies gilt insbesondere für Europa, wo an mehr als der Hälfte (29 von 55$)$ der laufenden Studien mehrere Länder beteiligt sind. Dabei ist die gesamte Zahl laufender CAR-TZellstudien in Deutschland seit 2017 fast um den Faktor 10 gestiegen. Deutschland und Frankreich sind innerhalb der Europäischen Union führend an CART-Studien beteiligt (27 bzw. 28 laufende Studien; - Abb. 2c,d ). Weltweit befinden sich mehr als die Hälfte der registrierten Studien in Phase 1, inzwischen aber auch fast 30 der über 650 registrierten Studi- en in Phase 3 oder Langzeituntersuchungen $(\bullet A b b .2 b)$. Letztere sind von großer Bedeutung, um besser zu verstehen, wie lange sich CAR-T-Zellen im Patienten aufhalten, dies sowohl im Hinblick auf etwaige Nebenwirkungen als auch auf ihre antitumorale Aktivität.

Die mit Abstand größte Anzahl klinischer Studien beruht auf CD19 als Zielantigen (• Abb. 3). Dies mag verwundern, da doch bereits 2 solcher CAR-TZellprodukte über eine Marktzulassung verfügen. Genau hier zeigt sich allerdings das Potenzial dieses therapeutischen Ansatzes, welches mit den beiden zugelassenen Arzneimitteln selbst für CD19 als Zielantigen noch lange nicht erschöpft ist. So stehen in laufenden Studien mit CD19-CAR-T-Zellen Fragen im Fokus, welche die Ausweitung der Indikationen, Vereinfachungen im Herstellungsprozess, Veränderungen im CAR- 

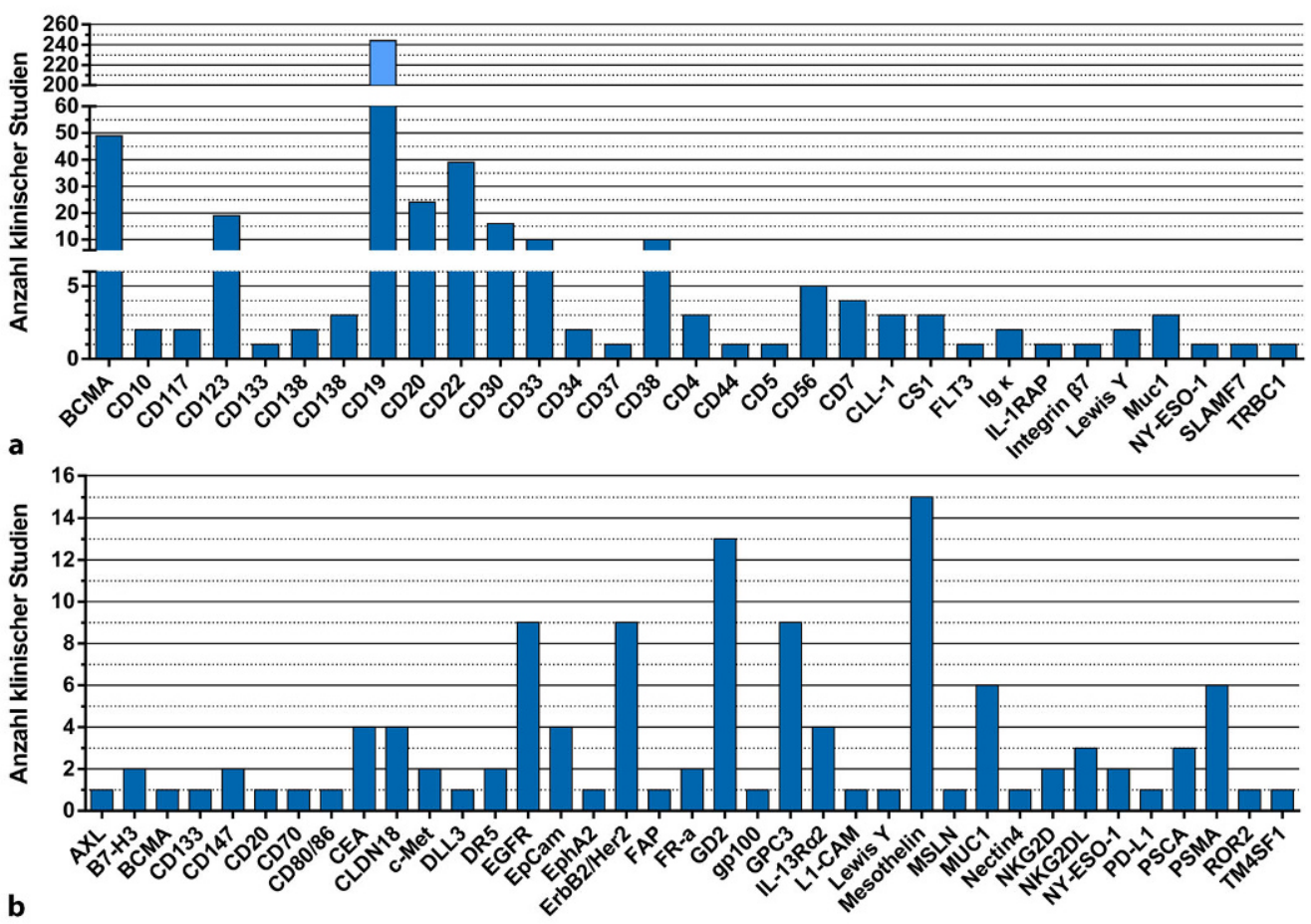

\begin{abstract}
Abb. $3<$ Antigene für hämatologische a und solide b Tumorerkrankungen die aktuell in CAR-T-Zellstudien untersucht werden. Die Angaben sind aus dem freiwilligen US-Register clinicaltrials.gov entnommen. Für 5 CAR-T-Zellstudien bei hämatologischen und 6 Studien bei soliden Tumorerkrankungen sind keine Angaben zum Antigen vorhanden
\end{abstract}

Design, die Art der verwendeten T-Zellsubtypen oder die Kombination verschiedener CARs mit Spezifität gegen mehrere Tumorantigene in einem Produkt betreffen.

Daneben werden in vielen laufenden Studien CAR-T-Zellen mit Spezifität für andere Zielmoleküle evaluiert. Dies betrifft nach wie vor insbesondere hämatologische Tumorerkrankungen. Hier befinden sich CAR-T-Zellen, die das Antigen BCMA (B-cell Maturation Antigen) erkennen, in fortgeschrittener klinischer Erprobung zur Behandlung des multiplen Myeloms [26]. Danach folgen CD30 (Hodgkin-Lymphom) sowie die B-Zellmarker CD123, CD22 und CD20 (- Abb. 3a). Letztere sind unter anderem als alternative Zielantigene zu CD19 in der Testung. Weil es bei der CAR-Therapie zu antigennegativen (z.B. CD19-negativen) Rückfällen kommen kann [27], sind die Erforschung und Verfügbarkeit mehrerer Marker für das gleiche Ziel von hoher Bedeutung für die Gewährleistung langfristig effektiver Therapie. Erste Studien untersuchen nun auch den Einsatz der CAR-Therapie bei T-Zelllymphomen, etwa über CD7. Hier müssen allerdings intelligente Maßnahmen er- griffen werden, um einen „Brudermord“ an den ebenfalls CD7-positiven CAR-TZellen zu verhindern [28].

Die Bekämpfung von soliden Tumoren stellt einen nächsten Schritt in der Anwendung von CAR-Zellen dar, der sich noch gänzlich in frühen klinischen Phasen befindet. Hier ergeben sich neue Herausforderungen für klinische Forscher, die bereits in dutzenden Studien untersucht werden (- Abb. 3b). So haben Studien zur Bekämpfung von Neuroblastomen mit gegen GD2-spezifischen CAR-T-Zellen zwar nur geringe Behandlungserfolge gezeigt, aber Einblicke in die zugrunde liegenden Mechanismen erlaubt. Demnach tragen eine unzureichende Persistenz der CAR-T-Zellen, nicht ausreichend selektive Tumormarker und immunsuppressive Tumormikroumgebungen zur bisher geringen Effektivität bei [29]. Trotz dieser Schwierigkeiten werden ermutigende Fortschritte gemacht: So hat eine klinische Studie zur Behandlung von Pleuramesotheliomen mit mesothelingerichteten CAR-T-Zellen in Kombination mit PD-1-Blockade die Sicherheit der Produktanwendung und erste Hinweise auf Effektivität ge- liefert [30]. Die CAR2BRAIN-Phase-1Studie in Frankfurt am Main wird zeigen, ob CAR-NK-Zellen gerichtet auf das Tumorantigen Her2/neu das Potenzial haben, den Herausforderungen der soliden Tumorumgebung im Kontext von Glioblastomen $\mathrm{zu}$ begegnen [31]. Die Gesamtzahl von CAR-NK-Studien mit aktuell 15 ist allerdings noch gering [32]. Dennoch werden die Ergebnisse dieser Studien in Fachkreisen mit Spannung erwartet.

\section{Hürden in der Anwendung und mögliche Lösungsansätze}

In den oben zusammengefassten klinischen Anwendungen werden sehr schwer erkrankte Patienten, die beispielsweise an aggressiven, gegenüber etablierten Arzneimitteln resistenten Leukämien oder Lymphomen leiden, mit CAR-TZellen behandelt. Die dabei erzielten Behandlungserfolge rechtfertigen die Hoffnung, dass die CAR-Therapie eine breit anwendbare Krebstherapie für viele Patienten werden könnte. Jedoch ist die klinische Anwendung von CAR-T-Zellen nicht ohne Probleme. Können diese adressiert werden, könnte die Nutzen- 
Risiko-Abwägung auch für Patienten in früheren Stadien der Erkrankung zugunsten der CAR-T-Therapie ausfallen.

\section{Nebenwirkungen}

CRS ist ein Sammelbegriff für ein Spektrum klinischer Symptome und Laborbefunde, die bei mit CAR-T-Zellen behandelten Patienten auftreten. Dazu gehören Fieber, niedriger Blutdruck, Hypoxie und neurologische Auffälligkeiten sowie deutlich erhöhte Level von Zytokinen im Serum. Wenngleich CRS verschiedener Schweregrade nicht nur bei Anti-CD19-CAR-Therapie auftritt, sondern auch im Kontext anderer Zielantigene beschrieben wurde, treten die meisten Fälle schweren Zytokinfreisetzungssyndroms bei der Behandlung hämatologischer Krankheitsbilder mit Anti-CD19CARs auf $[14,33]$. CRS tritt bei mehr als der Hälfte der mit Tisagenlecleucel oder Axicabtagene ciloleucel behandelten Patienten auf. Jedoch ist dabei die Inzidenz schwerer, medikamentöse Intervention erfordernder Fälle von CRS vom Produkt und der Indikation abhängig. So reichte die Frequenz schwerer Fälle in den relevanten Zulassungsstudien von $12 \%$ für Axicabtagene ciloleucel über $22 \%$ für Tisagenlecleucel bei Lymphomen bis zu $44 \%$ für Tisagenlecleucel bei Leukämien [19, 20, 34].

Zusätzlich ist bei der CAR-T-Behandlung von hämatologischen Krankheitsbildern Neurotoxizität beschrieben worden, die für wenige Patienten tödlich war [4, 14]. Zur Milderung schwerer Nebenwirkungen der CAR-T-Therapie werden Patienten mit Corticosteroiden, allein oder in Kombination mit dem antagonistischen Anti-IL6R-Antikörper Tocilizumab, behandelt. Während inzwischen genaue Protokolle für das Management sowohl von CRS als auch (der schlechter handhabbaren) Neurotoxizität existieren [34, 35], ist das molekulare Verständnis der Phänomene noch unzureichend, um für alle Fälle hocheffektive Interventionen $\mathrm{zu}$ definieren, die das therapeutische Potenzial der CAR-T-Zellen (im Gegensatz zu Corticosteroidbehandlung) erhalten.

Zur präklinischen Untersuchung von CRS und Neurotoxizität werden häu- fig Mausmodelle eingesetzt. Weil es gilt, die Wirkung menschlicher CARZellpräparate $\mathrm{zu}$ evaluieren, werden dafür komplexe, sogenannte humanisierte Mausmodelle verwendet. Aufgrund der hohen molekularen Spezifität von CARZellen muss ein dem menschlichen möglichst ähnlicher immunologischer Kontext geschaffen werden. Hierbei gilt es nicht nur, Zielzellen mit dem Zielantigen bereitzustellen, sondern auch, Reaktionen des murinen Immunsystems auf menschliche Zellen und - wichtiger - der implantierten menschlichen Immunzellen auf das murine Wirtsgewebe (Transplantat-gegen-Wirt-Reaktion, engl. Graft versus Host Disease, GvHD) zu verhindern bzw. hinauszuzögern, um die Aussagekraft des Modellsystems zu gewährleisten. Wie in $\bullet$ Abb. 4 veranschaulicht, werden dazu menschliche Blutstammzellen in durch genetische Manipulation immundefizient gemachte Mäuse transplantiert. In so humanisierten Mäusen können dann die Interaktionen zwischen menschlichen CARZellen und Tumorzellen sowie zwischen CAR-Zellen und anderen Immunzellen charakterisiert werden, um die dem CRS und der Neurotoxizität zugrunde liegenden Mechanismen zu erschließen. So konnten Experimente in mit adulten Blutstammzellen humanisierten NSG-Mäusen die Antitumoreffekte, das CRS und die Neurotoxizität von Anti-CD19-CAR-T-Zellen rekapitulieren. Interessanterweise konnte dabei Monozyten eine wichtige Rolle zugeschrieben werden und neben Interleukin-6 (IL-6) konnte IL-1 als Ursache für CRS und Neurotoxizität identifiziert werden [6]. Komplementär dazu sind Ergebnisse aus einem Modell auf Basis immundefizienter Mäuse ohne separate menschliche Immunrekonstitution, die den Ausstoß von IL-6, IL-1 und Stickoxid (NO) durch Makrophagen als kritische Regulatoren des CRS identifizierten [5].

Humanisierte Modelle sind hilfreich zur direkten Evaluierung von für die Behandlung von Menschen gemachten Zellpräparaten. Ihre komplexe Herstellung macht sie allerdings fragil in der Handhabung und teuer in der Herstellung. Zudem kann durch die hybride Natur der humanisierten Immunsyste- me nicht sichergestellt werden, dass die scheinbar wichtigen Schnittstellen zwischen den myeloiden und lymphoiden Komponenten des Immunsystems korrekt abgebildet sind. Ein weiterer Ansatz, der auch bei der präklinischen Evaluation von Axicabtagene ciloleucel verfolgt wurde [36], ist, ein zum menschlichen System analoges, komplett murines System zu verwenden. Dabei werden murine Antigene erkennende CARs auf murinen Zellen zur Bekämpfung von Tumorzellen murinen Ursprungs eingesetzt. Eine Angleichung an die klinische Situation kann etwa durch Bestrahlung der Mäuse oder Vorbehandlung mit Chemotherapeutika erfolgen. Letztere Strategie wurde verwendet, um den Einfluss von Katecholaminsignaling auf CRS in einem syngenen Mausmodell zu untersuchen [8].

Die der Neurotoxizität zugrunde liegenden Mechanismen sind unzureichend verstanden. Allerdings demonstrierte eine klinische Studie vor Kurzem, dass die Verwendung eines CAR mit ausschließlich menschlichen Sequenzen (im Gegensatz z.B. zu den in Axicabtagene ciloleucel und Tisagenlecleucel verwendeten, von der Maus abgeleiteten Antigenbindedomänen) in wesentlich geringeren Raten in Neurotoxizität resultierte [37]. Eine weitere Möglichkeit, adverse Effekte $\mathrm{zu}$ umgehen oder $\mathrm{zu}$ mindern, könnte in der Verwendung von CAR-NK-Zellen bestehen. Es ist vermutlich wegen ihrer von T-Zellen abweichenden Aktivierungsmechanismen - möglich, allogene NK-Zellen sicher adoptiv zu transferieren [16]. Eine neue klinische Phase-1/2-Studie zum Einsatz von Anti-CD19-CAR-NK-Zellen bei B-Zelltumoren fand keine Belege für CRS, Neurotoxizität oder GvHD bei der Transplantation partiell MHCinkongruenter und/oder killer-immunoglobulin-like-receptor-(KIR-)inkompatibler allogener CAR-NK-Zellen [38]. Zur eindeutigeren Beurteilung der Antitumoraktivität von CAR-NK-Zellen bedarf es allerdings weiterer Studien.

\section{Herstellung}

Eine zweite wesentliche Hürde für die breite klinische Anwendung von CAR- 


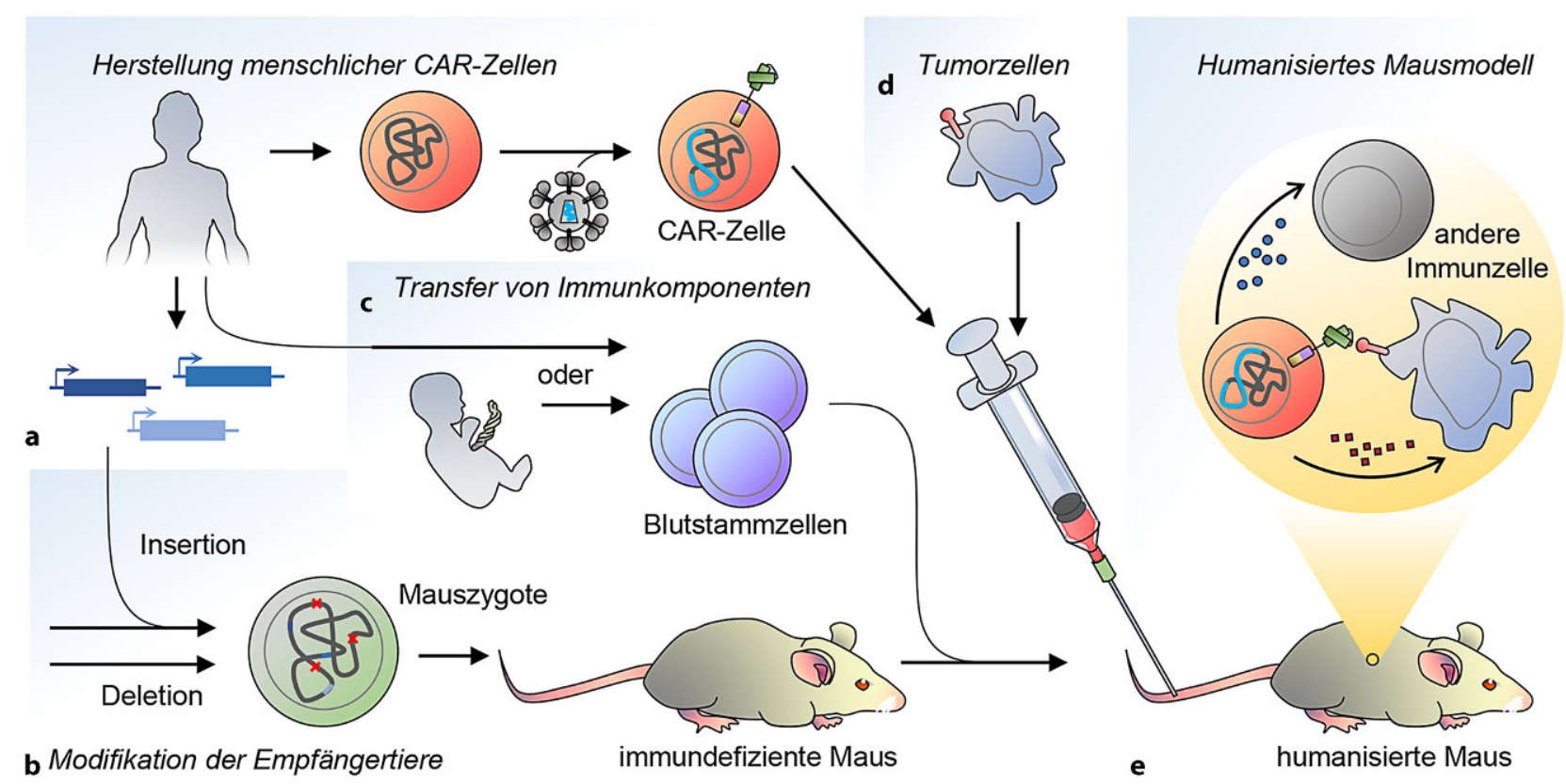

\begin{abstract}
Abb. 4 A Humanisierte Mausmodelle zur präklinischen Erforschung der Nebenwirkungen der CAR-Therapie. a CAR-Zellen werden aus der Leukapherese eines menschlichen Spenders hergestellt, zum Beispiel durch Behandlung mit lentiviralen Vektoren, und zum geeigneten Zeitpunkt in die humanisierte Maus eingebracht. b Durch Deletion kritischer Gene wird eine immundefiziente Maus generiert. Manche Modelle nutzen Mäuse, in deren Genom zusätzlich menschliche Gene eingefügt wurden, die das Wachstum menschlicher Immunzellen begünstigen. cDer immundefizienten Maus werden zelluläre menschliche Immunkomponenten implantiert. Hierbei kann es sich, abhängig vom Modell, z. B. um alle mononukleären Zellen oder CD34+-Blutstammzellen handeln, gewonnen aus dem peripheren Blut von Erwachsenen oder Nabelschnurblut. d Geeignete Tumorzellen werden der humanisierten Maus injiziert, z. B. die B-Zelllymphomalinie Nalm6. e Das komplette humanisierte Mausmodell bildet die Interaktion der menschlichen CAR-Zellen mit menschlichen Tumorzellen und anderen Immunzellen menschlichen Ursprungs nach und kann zur Untersuchung der antitumoralen Aktivität und ggf. Nebenwirkungen von CARTherapien genutzt werden
\end{abstract}

Zellen stellt die logistisch sehr aufwendige, wenig standardisierte, fehleranfällige und teure Herstellung dar. Aktuell werden CAR-T-Zellprodukte autolog, d.h. aus der Leukapherese von Patienten hergestellt. Dies verlangt nach gut an die jeweiligen Behandlungszentren angeschlossener Good-ManufacturingPractice-(GMP-)Infrastruktur mit Kapazitäten für individualisierte Bioprozesstechnik. Wohl auch als Folge von Faktoren wie der logistischen und technischen Schwierigkeit der Herstellung des Zellprodukts sind die Kosten für eine Behandlung mit kommerziellen CAR-T-Produkten prohibitiv hoch: Gemäß den Beschlüssen des Gemeinsamen Bundesauschusses zu Tisagenlecleucel und Axicabtagene ciloleucel liegen die Jahrestherapiekosten pro Patient bei ca. $320.000 €$ bzw. ca. $390.000 €[39$, 40]. Abhängig vom Therapiezentrum können sich die Jahrestherapiekosten basierend auf der krankenhausindividuellen Vereinbarung der Vergütung von neuen Untersuchungs- und Behandlungsmethoden (NUB) jedoch auch unter $300.000 €$ belaufen [41]. Durch einen Mangel an Standardisierung existiert eine erhebliche Heterogenität unter den Verfahren, die weltweit zur Produktion autologer CAR-T-Produkte eingesetzt werden.

Gemeinsam ist den Verfahren hohe Variabilität innerhalb des Prozesses, zum Beispiel betreffend Zellzahlen, Zellwachstum und Transduktionseffizienz. Die „offene“ Manipulation der Zellen im Verlauf des Prozesses macht diesen anfällig für Kontamination und andere Handhabungsfehler. Für viele Schritte, zum Beispiel die Zellisolierung, die Aktivierung der Zellen oder die Transduktion, ist unklar, welche Technik optimale Ergebnisse erzielt $[17,18]$. Ein wichtiger Schritt hin zu einer konsistenteren (und damit kosteneffektiveren) Herstellung von CAR-T-Zellen könnte die Automatisierung des Herstellungsprozesses sein. Mit ihr könnten die mit manuellen Arbeitsschritten verbundenen Risiken und Variationen eliminiert werden. Während automatisierte Zellkulturplattformen der ersten Generation - zur Minimierung der von manuellen Schritten ausgehenden Prozessvariabilität einzelne Abschnitte eines Bioprozesses automatisieren, können automatisierte Zellkulturplattformen der zweiten Generation als Zusammenfassung eines ganzen personalisierten Bioprozesses in einem Gerät verstanden werden, die vollautomatische Produktion z. B. eines CAR-T-Zellprodukts ermöglichen [42].

Die durchschnittliche Herstellungsdauer von autologen CAR-T-Zellprodukten beträgt 12 Tage (7-22 Tage; [17]). Bei aggressiven Krankheitsbildern wäre allerdings die schnellstmögliche Be- 
handlung im Sinne des Patienten, auch weil größere Tumorlast im Knochenmark mit schwererem CRS assoziiert wurde [34]. Zudem können der schlechte Zustand des Patienten zum Zeitpunkt der Spende und der damit assoziierte suboptimale Zustand der Leukapherese hinsichtlich Viabilität und Zellzahlen die Produktion des CAR-T-Zellprodukts erheblich erschweren. Zusätzlich besteht bei Zellspenden aus leukämischen Patienten das Risiko der Transduktion und Reinfusion leukämischer Blasten. Ein solcher Fall von CAR-Blasten mit tödlichem Ausgang ist aus einer Phase-1Studie zu Tisagenlecleucel bekannt [43]. Die Verwendung allogener, d.h. von gesunden Spendern abgeleiteter CAR-TZellen könnte diese Probleme umgehen helfen. Allogene CAR-T-Zellen würden auf Basis „fitterer" Zellen produziert und wären damit konsistenter und einfacher herzustellen. Sie würden sich im Vornherein herstellen lassen und wären zum klinisch sinnvollsten Zeitpunkt einsetzbar, wenn nötig mit minimaler Verzögerung zwischen Behandlungsentscheidung und Behandlung.

Ähnlich wie bei der Spende von Blutstammzellen [44] gilt es allerdings, das Risiko durch Implantation fremder Immunzellen ausgelöster Komplikationen einzuschätzen. $\mathrm{Zu}$ diesen zählen GvHD und durch den Wirt vermittelte AbstoBung der transplantierten Zellen (engl. Host-mediated Rejection). Strategien zur Kontrolle dieser Effekte beinhalten die genetische Ablation der Expression von T-Zellrezeptoren (TCRs) und MHCMolekülen auf Spenderzellen. Des Weiteren können Spenderzellen (z. B. durch die Entfernung von CD52) so modifiziert werden, dass sie - im Gegensatz zu Wirtsimmunzellen - resistent gegenüber Lymphodepletion sind. Entsprechende klinische Studien laufen [45].

Ebenfalls könnten nichtvirale Transferstrategien dazu beitragen, die Produktion von CAR-Zellen ex vivo zu verbessern. CAR-Transgene können z.B. als Plasmide mittels Elektroporation in die Immunzelle eingebracht und mittels Transposasen in deren Genom integriert werden oder in Form von synthetischer mRNA transferiert werden, die eine zeitlich begrenzte CAR-Expression er- möglicht. Vorteile gegenüber viralem Transfer sind dabei niedrigere Kosten und ein geringeres Risiko von insertioneller Onkogenese [18]. Gegenstand aktueller Forschung ist ebenfalls die Verwendung von sogenannten Genscheren wie der CRISPR/Cas9-Technologie, um eine präzise Veränderung des Immunzellgenoms zu erreichen, z. B. die gezielte Insertion des CAR-Gens in einen T-Zellrezeptorlokus [46].

Zukünftig könnte ein alternativer Ansatz in der Generierung von CAR-T-Zellen in vivoliegen, also direkt im Patienten. Dafür müssten lediglich Vektoren injiziert werden, welche die für den CAR codierende Genkassette spezifisch in die T-Zellen einbringen. Diese Vorgehensweise befindet sich allerdings noch in der präklinischen Entwicklung. In einem Ansatz wurden mithilfe von CD3-targetierten Nanopartikeln CAR-T-Zellen in Mäusen generiert [47]. In einer weiteren Arbeit konnten humane CAR-T-Zellen mit CD8-targetierten lentiviralen Vektoren in humanisierten Mäusen erzeugt werden [7, 48]. Ob solche In-vivo-Ansätze tatsächlich das Potenzial, haben die komplexe Ex-vivo-Herstellung von CART-Zellen zu umgehen, wird sich an Großtiermodellen erweisen müssen.

\section{Fazit und Ausblick}

Mit der CAR-Therapie beginnt ein neues Kapitel der Krebstherapie. Trotz der rapiden Entwicklungen in der Erforschung und Anwendung von CARTherapien und ihrer beeindruckenden Erfolge ist Geduld angebracht. CARProdukte gehören zur bisher kleinen Gruppe der Arzneimittel für fortgeschrittene Therapien (engl. Advanced Therapy Medicinal Products, ATMPs) und sind damit einzigartig unter den Krebstherapien. Dementsprechend klein ist die Erfahrungsbasis, nicht nur für die hier besprochenen technischen Aspekte ihrer Herstellung und Anwendung, sondern auch für ihre Zulassung durch die entsprechenden regulatorischen Institutionen. Das durch die Studienlage unterstrichene globale Interesse, CART-Therapien voranzubringen und breit nutzbar zu machen, bereitet den Boden für die Erforschung, Zulassung und
Anwendung von CAR-ATMPs nicht nur für weit fortgeschrittene Tumorerkrankungen, sondern auch für eine Bandbreite von anderen Indikationen, von Infektionskrankheiten wie HIV [49] über Autoimmunkrankheiten bis hin zur allogenen Transplantation [50]. So könnte die Krebstherapie mit chimären Antigenrezeptoren als Türöffner für neue Entwicklungen in der Zelltherapie fungieren, die das Potenzial haben, nicht nur die Onkologie nachhaltig zu verändern.

\section{Korrespondenzadresse}

Prof. Dr. Christian J. Buchholz

Paul-Ehrlich-Institut

Paul-Ehrlich-Str. 51-59, 63225 Langen,

Deutschland

christian.buchholz@pei.de

Förderung. Diese Arbeit wurde durch Fördermittel des Bundesgesundheitsministeriums (BMG) unterstützt (Az. 03292364).

Funding. Open Access funding enabled and organized by Projekt DEAL.

\section{Einhaltung ethischer Richtlinien}

Interessenkonflikt. A. Michels, J. Hartmann und C.J. Buchholz geben an, dass kein Interessenkonflikt besteht.

Für diesen Beitrag wurden von den Autoren keine Studien an Menschen oder Tieren durchgeführt. Für die aufgeführten Studien gelten die jeweils dort angegebenen ethischen Richtlinien.

Open Access. Dieser Artikel wird unter der Creative Commons Namensnennung 4.0 International Lizenz veröffentlicht, welche die Nutzung, Vervielfältigung, Bearbeitung, Verbreitung und Wiedergabe in jeglichem Medium und Format erlaubt, sofern Sie den/die ursprünglichen Autor(en) und die Quelle ordnungsgemäß nennen, einen Link zur Creative Commons Lizenz beifügen und angeben, ob Änderungen vorgenommen wurden.

Die in diesem Artikel enthaltenen Bilder und sonstiges Drittmaterial unterliegen ebenfalls der genannten Creative Commons Lizenz, sofern sich aus der Abbildungslegende nichts anderes ergibt. Sofern das betreffende Material nicht unter der genannten Creative Commons Lizenz steht und die betreffende Handlung nicht nach gesetzlichen Vorschriften erlaubt ist, ist für die oben aufgeführten Weiterverwendungen des Materials die Einwilligung des jeweiligen Rechteinhabers einzuholen.

Weitere Details zur Lizenz entnehmen Sie bitte der Lizenzinformation auf http://creativecommons.org/ licenses/by/4.0/deed.de. 


\section{Literatur}

1. Statistisches Bundesamt (2020) Todesursachen in Deutschland. Todesursachen. https:// www.destatis.de/DE/Themen/GesellschaftUmwelt/Gesundheit/Todesursachen/ inhalt. html\#sprg234240.Zugegriffen:3. Apr.2020

2. Salles G, Barrett M, Foà R et al (2017) Rituximab in B-cell hematologic malignancies: a review of 20 years of clinical experience. Adv Ther 34(10):2232-2273. https://doi.org/10.1007/ s12325-017-0612-X

3. Wei SC, Duffy CR, Allison JP (2018) Fundamental mechanisms of immune checkpoint blockade therapy. Cancer Discov 8(9):1069-1086. https:// doi.org/10.1158/2159-8290.CD-18-0367

4. Hartmann J, Schüßler-Lenz $M$, Bondanza $A$, Buchholz CJ (2017) Clinical development of CAR T cells-Challenges and opportunities in translating innovative treatment concepts. EMBO Mol Med 9(9):1183-1197. https://doi.org/10. 15252/emmm.201607485

5. Giavridis T, van der Stegen SJC, Eyquem J, Hamieh M, Piersigilli A, Sadelain M (2018) CAR $T$ cell-induced cytokine release syndrome is mediated by macrophages and abated by IL- 1 blockade. Nat Med. https://doi.org/10.1038/ s41591-018-0041-7

6. Norelli M, Camisa B, Barbiera G et al (2018) Monocyte-derived IL-1 and IL-6 are differentially required for cytokine-release syndrome and neurotoxicity due to CAR T cells. Nat Med. https:// doi.org/10.1038/s41591-018-0036-4

7. Pfeiffer A, Thalheimer FB, Hartmann Setal (2018) In vivo generation of human CD19-CART cells results in B-cell depletion and signs of cytokine release syndrome. EMBO Mol Med 11(10):e9158. https:// doi.org/10.15252/emmm.201809158

8. Staedtke V, Bai RY, Kim K et al (2018) Disruption of a self-amplifying catecholamine loop reduces cytokine release syndrome. $\mathrm{Na}$ ture 564(7735):273-277. https://doi.org/10.1038/ s41586-018-0774-y

9. Duan H, Huang H, Jing G (2019) An antibody Fab fragment-based chimeric antigen receptor could efficiently eliminate human thyroid cancer cells. J Cancer 10(8):1890-1895. https://doi.org/ 10.7150/jca.30163

10. Balakrishnan A, Rajan A, Salter Al et al (2019) Multispecific targeting with synthetic ankyrin repeat motif chimeric antigen receptors. Clin Cancer Res. https://doi.org/10.1158/1078-0432. CCR-19-1479

11. You $F$, Wang $Y$, Jiang $L$ et al (2019) A novel CD7 chimeric antigen receptor-modified NK-92MI cell line targeting T-cell acute lymphoblastic leukemia. Am J Cancer Res 9(1):64-78

12. Guedan S, Calderon H, Posey AD, Maus MV (2019) Engineering and design of chimeric antigen receptors. Mol Ther Methods Clin Dev 12:145-156. https://doi.org/10.1016/j.omtm.2018.12.009

13. Chmielewski M, Hombach AA, Abken H (2014) Of CARs and TRUCKs: chimeric antigen receptor (CAR) T cells engineered with an inducible cytokine to modulate the tumor stroma. Immunol Rev 257(1):83-90. https://doi.org/10.1111/imr.12125

14. June $\mathrm{CH}$, Sadelain M (2018) Chimeric antigen receptor therapy. N Engl J Med 379(1):64-73. https://doi.org/10.1056/NEJMra1706169

15. Morvan MG, Lanier LL (2016) NK cells and cancer: you can teach innate cells new tricks. Nat Rev Cancer 16(1):7-19. https://doi.org/10.1038/nrc. 2015.5
16. Miller JS, Soignier Y, Panoskaltsis-Mortari A et al (2005) Successful adoptive transfer and in vivo expansion of human haploidentical NK cells in patients with cancer. Blood 105(8):3051-3057. https://doi.org/10.1182/blood-2004-07-2974

17. Roddie C, O'Reilly M, Dias Alves Pinto J, Vispute $K$ Lowdell M (2019) Manufacturing chimeric antigen receptorT cells: issues and challenges. Cytotherapy 21(3):327-340. https://doi.org/10.1016/j.jcyt. 2018.11.009

18. Vormittag P, Gunn R, Ghorashian S, Veraitch FS (2018) A guide to manufacturing CAR T cell therapies. Curr Opin Biotechnol 53:164-181. https://doi.org/10.1016/j.copbio.2018.01.025

19. European Medicines Agency (2018) Yescarta: EPAR public assessment report. https://www.ema. europa.eu/en/documents/assessment-report/ yescarta-epar-public-assessment-report_en.pdf. Zugegriffen: 10.Apr. 2020

20. European Medicines Agency (2018) Kymriah: EPAR public assessment report. https://www. ema.europa.eu/documents/assessment-report/ kymriah-epar-public-assessment-report_en.pdf Zugegriffen: 10.Apr. 2020

21. Elsallab M, Levine BL, Wayne AS, Abou-ElEnein M (2020) CAR T-cell product performance in haematological malignancies before and after marketing authorisation. Lancet Oncol 21(2):e104-e116. https://doi.org/10.1016/S14702045(19)30729-6

22. Jacobson CA, Hunter B, Armand P et al (2018) Axicabtagene ciloleucel in the real world: outcomes and predictors of response, resistance and toxicity. Blood 132(Supplement 1):92. https://doi.org/10. 1182/blood-2018-99-117199

23. Nastoupil LJ, Jain MD, Spiegel JY et al (2018) Axicabtagene Ciloleucel (Axi-cel) CD19 chimeric antigen receptor (CAR) T-cell therapy for relapsed/ refractory large B-cell lymphoma: real world experience. Blood 132(Supplement 1):91. https:// doi.org/10.1182/blood-2018-99-114152

24. Riedell PA, Bishop MR (2020) Safety and efficacy of axicabtagene ciloleucel in refractory large B-cell lymphomas. Ther Adv Hematol 11:2040620720902899. https://doi.org/10.1177/ 2040620720902899

25. U.S. National Library of Medicine (2020) ClinicalTrials.gov. https://clinicaltrials.gov/ct2/home. Zugegriffen: 10.Apr. 2020

26. Sellner L, Fan F, Giesen N et al (2020) B-cell maturation antigen-specific chimeric antigen receptor $T$ cells for multiple myeloma: clinical experience and future perspectives. Int J Cancer. https://doi.org/10.1002/ijc.33002

27. Jackson HJ, Brentjens RJ (2015) Overcoming antigen escape with CAR T-cell therapy. Cancer Discov 5(12):1238-1240. https://doi.org/10.1158/ 2159-8290.CD-15-1275

28. Cooper ML, Choi J, Staser K et al (2018) An "off-the-shelf" fratricide-resistant CAR-T for the treatment of $\mathrm{T}$ cell hematologic malignancies. Leukemia 32(9):1970-1983. https://doi.org/10. 1038/s41375-018-0065-5

29. Richards RM, Sotillo E, Majzner RG (2018) CART cell therapy for neuroblastoma. Front Immunol 9:2380. https://doi.org/10.3389/fimmu.2018.02380

30. Adusumilli PS, Zauderer MG, Rusch VW et al (2019) Regional delivery of mesothelin-targeted CAR T cells for pleural cancers: Safety and preliminary efficacy in combination with anti-PD-1 agent. J Clin Oncol 37(15_suppl):2511. https://doi.org/ 10.1200/JCO.2019.37.15_suppl.2511

31. Burger MC, Zhang C, Harter PN et al (2019) CAR-engineered NK cells for the treatment of glioblastoma: turning innate effectors into precision tools for cancer immunotherapy. Front Immunol 10:2683. https://doi.org/10.3389/ fimmu.2019.02683

32. Kloess S, Kretschmer A, Stahl L, Fricke S, Koehl U (2019) CAR-expressing natural killer cells for cancer retargeting. Transfus Med Hemother 46(1):4-13. https://doi.org/10.1159/000495771

33. Thakar MS, Kearl TJ, Malarkannan S (2020) Controlling cytokine release syndrome to harness the full potential of CAR-based cellular therapy. Front Oncol. https://doi.org/10.3389/fonc.2019. 01529

34. Chou CK, Turtle CJ (2020) Assessment and management of cytokine release syndrome and neurotoxicity following CD19 CAR-T cell therapy. Expert Opin Biol Ther. https://doi.org/10.1080/ 14712598.2020.1729735

35. Rivera AM, May S, Lei M et al (2020) CAR T-cellassociated neurotoxicity: current management and emerging treatment strategies. Crit Care Nurs Q 43(2):191-204. https://doi.org/10.1097/ CNQ.0000000000000302

36. Kochenderfer JN, Yu Z, Frasheri D, Restifo NP, Rosenberg SA (2010) Adoptive transfer of syngeneic $T$ cells transduced with a chimeric antigen receptor that recognizes murine CD19 can eradicate lymphoma and normal B cells. Blood 116(19):3875-3886. https://doi.org/10. 1182/blood-2010-01-265041

37. Brudno JN, Lam N, Vanasse D et al (2020) Safety and feasibility of anti-CD19 CAR T cells with fully human binding domains in patients with B-cell lymphoma. Nat Med 26(2):270-280. https://doi. org/10.1038/s41591-019-0737-3

38. Liu E, Marin D, Banerjee P et al (2020) Use of CARTransduced natural killer cells in CD19-positive lymphoid tumors. N Engl J Med 382(6):545-553. https://doi.org/10.1056/NEJMoa1910607

39. Gemeinsamer Bundesausschuss (2020) Arzneimittel-Richtlinie/Anlage XII: Tisagenlecleucel (diffus großzelliges B-Zell-Lymphom) - Gemeinsamer Bundesausschuss. https://www.g-ba.de/ beschluesse/3700/.Zugegriffen:6. Mai 2020

40. Gemeinsamer Bundesausschuss (2020) Nutzenbewertungsverfahren zum Wirkstoff AxicabtagenCiloleucel (Diffus großzelliges B-Zell-Lymphom) Gemeinsamer Bundesausschuss. https://www.gba.de/bewertungsverfahren/nutzenbewertung/ 408/\#beschluesse. Zugegriffen: 6. Mai 2020

41. Charité - Universitätsmedizin Berlin (2020) DRGEntgelttarif für Krankenhäuser im Anwendungsbereich des KHEntgG und Pflegekostentarif im Anwendungsbereich der BPflV sowie Unterrichtung des Patienten gemäß § 8 KHEntgG / §14 BPfIV. Entgelte 01.08.2020. https://www.charite. de.Zugegriffen: 18. Aug. 2020

42. Moutsatsou P, Ochs J, Schmitt RH, Hewitt CJ, Hanga MP (2019) Automation in cell and gene therapy manufacturing: from past to future. Biotechnol Lett 41(11):1245-1253. https://doi. org/10.1007/s10529-019-02732-z

43. Ruella M, Xu J, Barrett DM et al (2018) Induction of resistance to chimeric antigen receptor $T$ cell therapy by transduction of a single leukemic $B$ cell. Nat Med 10(24):1499-1503. https://doi.org/ 10.1038/s41591-018-0201-9

44. Petersdorf EW (2017) Role of major histocompatibility complex variation in graft-versushost disease after hematopoietic cell transplantation. F1000Res. https://doi.org/10.12688/ f1000research.10990.1 


\section{Leitthema}

45. Qasim W (2019) Allogeneic CART cell therapies for leukemia. Am J Hematol 94(S1):S50-S54. https:// doi.org/10.1002/ajh.25399

46. Eyquem J, Mansilla-Soto J, Giavridis T et al (2017) Targeting a CAR to the TRAC locus with CRISPR/Cas9 enhances tumour rejection. Nature 543(7643):113-117. https://doi.org/10. 1038/nature21405

47. Smith TT, Stephan SB, Moffett HF et al (2017) In situ programming of leukaemia-specific $T$ cells using synthetic DNA nanocarriers. Nat Nanotechnol 8(12):813-820. https://doi.org/10.1038/nnano. 2017.57

48. Agarwal S, Weidner T, Thalheimer FB, Buchholz CJ (2019) In vivo generated human CART cells eradicate tumor cells. Oncolmmunology 12(8):e1671761. https://doi.org/10.1080/2162402X.2019.1671761

49. Herzig E, Kim KC, Packard TA et al (2019) Attacking latent HIV with convertibleCAR-T cells, a highly adaptable killing platform. Cell 179(4):880-894.e10. https://doi.org/10.1016/j. cell.2019.10.002

50. Maldini C, Ellis G, Riley JL (2018) CAR T cells for infection, autoimmunity and allotransplantation. Nat Rev Immunol 18(10):605-616. https://doi.org/ 10.1038/s41577-018-0042-2 